C. Park and D. Skoug

Nagoya Math. J.

Vol. 110 (1988), 151-162

\title{
THE FEYNMAN INTEGRAL OF QUADRATIC POTENTIALS DEPENDING ON $n$ TIME VARIABLES
}

\author{
CHULL PARK AND DAVID SKOUG ${ }^{1}$
}

\section{§1. Introduction}

Let $C_{1}[0, T]$ denote (one-parameter) Wiener space; that is the space of continuous functions $x$ on $[0, T]$ such that $x(0)=0$. In a recent expository essay [21], Nelson calls attention to some functions on Wiener space which were discussed in the book of Feynman and Hibbs [13, section 3-10] and in Feynman's original paper [12, section 13]. These functions have the form

$$
G(x)=\exp \left\{\int_{0}^{T} \int_{0}^{T} W\left(s_{1}, s_{2} ; x\left(s_{1}\right), x\left(s_{2}\right)\right) d s_{1} d s_{2}\right\} .
$$

Feynman obtained such functions by integrating out the oscillator coordinates in a system involving a harmonic oscillator interacting with a particle moving in a potential. Further functions like (1.1) but involving multiple integrals of more dimensions than two arise when more particles are involved; the study of such functions is the topic of this paper. In particular we consider the case where the function $W:[0, T]^{n} \times R^{n} \rightarrow C$ is quadratic in the space variables.

In [8], Chang, Johnson and Skoug consider functions on Wiener space of the form

$$
F(x)=\exp \left\{-\int_{0}^{T} \int_{0}^{T}\left\langle A\left(s_{1}, s_{2}\right)\left(x\left(s_{1}\right), x\left(s_{2}\right)\right),\left(x\left(s_{1}\right), x\left(s_{2}\right)\right)\right\rangle d s_{1} d s_{2}\right\}
$$

where $\left\{A\left(s_{1}, s_{2}\right)=\left(a_{i j}\left(s_{1}, s_{2}\right)\right):\left(s_{1}, s_{2}\right) \in[0, T]^{2}\right\}$ is a commutative family of 2 by 2 real, symmetric, nonnegative definite matrices with their eigenvalues $p_{1}\left(s_{1}, s_{2}\right)$ and $p_{2}\left(s_{1}, s_{2}\right)$ having square roots which are of bounded variation on the rectangle $[0, T]^{2}$. They showed that such functions $F$ are in the Banach algebra $S$ of functions on Wiener space which was introduced by

Received October 7, 1986.

Revised February 24, 1987.

${ }^{1}$ Research partially supported by NSF Grant \#DMS-8403197. 
Cameron and Storvick [6]. In particular the functions $F$ are analytic Feyman integrable.

In section 3 of this paper we extend these results to functions of the form

$$
\begin{aligned}
F(x)= & \exp \left\{-\int_{0}^{T} \cdots \int_{0}^{T}\left\langle A\left(s_{1}, \cdots, s_{n}\right)\left(x\left(s_{1}\right), \cdots, x\left(s_{n}\right)\right),\right.\right. \\
& \left.\left.\left(x\left(s_{1}\right), \cdots, x\left(s_{n}\right)\right)\right\rangle d s_{1} \cdots d s_{n}\right\} .
\end{aligned}
$$

Perhaps more importantly we are able to substantially relax the requirements on the eigenvalues $p_{j}\left(s_{1}, \cdots, s_{n}\right)$. In this paper we simply require that the eigenvalues $p_{j}\left(s_{1}, \cdots, s_{n}\right)$ be in $L_{1}\left([0, T]^{n}\right)$ rather than requiring that their square roots be of bounded variation on $[0, T]^{n}$, a rather difficult condition to verify for $n>1$. In particular this permits us to considerably simplify the hypothesis of several theorems and corollaries.

In section 4 we extend the results of section 3 to $\nu$-dimensional Wiener space $C_{1}^{\nu}[0, T]=C_{1}[0, T] \times \cdots \times C_{1}[0, T]$ ( $\nu$ times). The formulations in [6] and [19] are $\nu$-dimensional with $n=1$ (one time parameter) while in [8], $\nu$ is 1 with $n=2$. In this section we let both $n$ and $\nu$ be arbitrary positive integers. In [8] the matrices $A\left(s_{1}, s_{2}\right)$ are 2 by 2 and involve two time parameters. In [19] the matrices $A(s)$ are $\nu$ by $\nu$ and involve one time parameter. In this section the matrices $A\left(s_{1}, \cdots, s_{n}\right)$ are of dimension $n \nu$ by $n \nu$ and involve $n$ time parameters. Of course, it is much more difficult to increase the number of time parameters than to handle more position dimensions.

We establish several corollaries which show that the Banach algebra $S$ contains a broad class of functions involving multiple integrals of potentials depending on $n$ time parameters. We also show that these functions are in Albeverio and Høegh-Krohn's space $\mathscr{F}(H)$ of Fresnel integrable functions [1-3].

It is interesting to note that while the statements of our results involve only the one-parameter Wiener process, the proofs heavily involve the $n$-parameter Wiener process in a most natural way. In particular we use a stochastic integration formula, see equation (2.9) below, involving a mix of one-parameter Wiener space and n-parameter Wiener space.

For some related work other than [8] and [19], see a recent paper by Elworthy and Truman [11], as well as a 1973 paper by Cameron and Storvick [5]. 


\section{§ 2. Preliminaries}

Let $n$ be a positive integer and let $Q=[0, T]^{n}$. Let $C_{n}=C_{n}(Q)$ denote $n$-parameter Wiener space; that is the space of continuous functions $f$ on $Q$ such that $f\left(0, s_{2}, \cdots, s_{n}\right)=f\left(s_{1}, 0, s_{3}, \cdots, s_{n}\right)=\cdots=f\left(s_{1}, \cdots, s_{n-1}, 0\right)$ $=0$ for all $\left(s_{1}, \cdots, s_{n}\right)$ in $Q$. We let $m_{n}$ denote Wiener measure on $C_{n}(Q)$ and we denote the Wiener integral of a measurable function $F: C_{n}(Q) \rightarrow$ $C$ by

$$
\int_{C_{n}(Q)} F(f) d m_{n}(f)
$$

Next we give the definition of the Paley-Wiener-Zygmund (P.W.Z.) integral for functions of $n$ variables. Let $\left\{\phi_{j}\right\}$ be a complete orthonormal $(\mathrm{CON})$ set of functions of bounded variation in the sense of Hardy-Krause [4] on $Q=[0, T]^{n}$. For $g$ in $L_{2}(Q)$, let

$$
g_{m}\left(s_{1}, \cdots, s_{n}\right)=\sum_{j=1}^{m}\left(g, \phi_{j}\right) \phi_{j}\left(s_{1}, \cdots, s_{n}\right) .
$$

The P.W.Z. integral is defined by the formula

$$
\int_{Q} g\left(s_{1}, \cdots, s_{n}\right) \tilde{d}_{n} f\left(s_{1}, \cdots, s_{n}\right)=\lim _{m \rightarrow \infty} \int_{Q} g_{m}\left(s_{1}, \cdots, s_{n}\right) d f\left(s_{1}, \cdots, s_{n}\right)
$$

for all $f$ in $C_{n}(Q)$ for which the limit exists. (In the case $n=1$, we denote the P.W.Z. integral by $\int_{0}^{T} g(s) \tilde{d} x(s)$.)

We need the concept of scale-invariant measurability [17, 7] in order to state various properties of the P.W.Z. integral. A subset $A$ of $C_{n}(Q)$ is said to be scale-invariant measurable provided $\rho A$ is Wiener measurable for every $\rho>0$, and a scale-invariant measurable set $N$ is said to be scaleinvariant null provided $m_{n}(\rho N)=0$ for every $\rho>0$. A property that holds except on a scale-invariant null set is said to hold scale-invariant almost everywhere (s-a.e.)

Next we state some useful facts about the P.W.Z. integral.

(2.3) For each $g$ in $L_{2}(Q)$, the P.W.Z. integral $\int_{Q} g \tilde{d}_{n} f$ exists for s-a.e. $f$ in $C_{n}(Q)$.

(2.4) The P.W.Z. integral $\int_{Q} g \tilde{d}_{n} f$ is essentially independent of the CON set $\left\{\phi_{j}\right\}$. 
(2.5) If $g$ is of bounded variation on $Q$, then the P.W.Z. integral $\int_{Q} g \tilde{d}_{n} f$ is s-a.e. equal to the Riemann Stieltjes integral $\int_{Q} g d f$.

(2.6) The P.W.Z. integral has the usual linearity properties.

(2.7) The map sending $g$ in $L_{2}(Q)$ to the function $F_{g}$ on $C_{n}(Q)$ given by $F_{g}(f)=\int_{Q} g \tilde{d}_{n} f$ is an isometric isomorphism of $L_{2}(Q)$ into $L_{2}\left(C_{n}(Q), m_{n}\right)$. Moreover, $\int_{C_{n}(Q)} \exp \left\{i \int_{Q} g \tilde{d}_{n} f\right\} \mathrm{dm}_{n}(f)=\exp \left\{-\frac{1}{2}\|g\|_{2}^{2}\right\}$.

(2.8) The sequence $\left\{\int_{Q} g_{m} d f\right\}, g_{m}$ given by (2.1) above, considered as a function of $f$, converges in $L_{2}\left(C_{n}(Q), m_{n}\right)$ mean to $\int_{Q} g \tilde{d}_{n} f$.

Finally we give a stochastic integration formula involving a mix of one-parameter Wiener space and $n$-parameter Wiener space that follows quite easily from a Fubini Theorem for multi-parameter P.W.Z. integrals that we recently established. This formula plays a major role in the proof of our main result, Theorem 3.1 below.

TheOREm 2.1. Let $n$ be a positive integer, let $Q=[0, T]^{n}$ and let $h \in$ $L_{2}(Q)$. For $k=1,2, \cdots, n$ let $E_{k}\left(s_{k}\right)=[0, T]^{k-1} \times\left[s_{k}, T\right] \times[0, T]^{n-k}$. Then for $k=1,2, \cdots, n$

$$
\begin{aligned}
& \int_{Q} h\left(s_{1}, \cdots, s_{n}\right) x\left(s_{k}\right) \tilde{d}_{n} f\left(s_{1}, \cdots, s_{n}\right) \\
& \quad=\int_{0}^{T}\left[\int_{E_{k}\left(s_{k}\right)} h\left(t_{1}, \cdots, t_{n}\right) \tilde{d}_{n} f\left(t_{1}, \cdots, t_{n}\right)\right] \tilde{d} x\left(s_{k}\right)
\end{aligned}
$$

for $m_{1} \times m_{n}$ - a.e. $(x, f) \in C_{1}[0, T] \times C_{n}(Q)$.

Proof. By use of the Fubini Theorem we obtain:

$$
\begin{aligned}
\int_{Q} h\left(s_{1}, \cdots, s_{n}\right) x\left(s_{k}\right) \tilde{d}_{n} f\left(s_{1} \cdots, s_{n}\right) \\
\quad=\int_{Q} h\left(s_{1}, \cdots, s_{n}\right)\left[\int_{0}^{T} \chi_{\left[0, s_{k}\right]}(u) \tilde{d} x(u)\right] \tilde{d}_{n} f\left(s_{1}, \cdots, s_{n}\right) \\
\quad=\int_{0}^{T}\left[\int_{Q} h\left(s_{1}, \cdots, s_{n}\right) \chi_{[u, T]}\left(s_{k}\right) \tilde{d}_{n} f\left(s_{1}, \cdots, s_{n}\right)\right] \tilde{d} x(u) \\
\quad=\int_{0}^{T}\left[\int_{E_{k}(u)} h\left(s_{1}, \cdots, s_{n}\right) \tilde{d}_{n} f\left(s_{1}, \cdots, s_{n}\right)\right] \tilde{d} x(u) .
\end{aligned}
$$




\section{§3. Quadratic potentials involving $n$-dimensional integrals}

First we define the Banach algebra $S$ mentioned in the introduction. Let $M=M\left(L_{2}[0, T]\right)$ be the collection of complex-valued countably additive measures on $\mathscr{B}=\mathscr{B}\left(L_{2}[0, T]\right)$, the Borel class of $L_{2}[0, T] . \quad M$ is a Banach algebra under the total variation norm where convolution is taken as the multiplication. The Banach algebra $S$ consists of functions $F$ on $C_{1}[0, T]$ expressible in the form

$$
F(x)=\int_{L_{2}[0, T]} \exp \left\{i \int_{0}^{T} g(s) \tilde{d} x(s)\right\} d \sigma(g)
$$

for s-a.e. $x$ in $C_{1}[0, T]$, where $\sigma$ is an element of $M$. Cameron and Storvick show that the correspondence $\sigma \rightarrow F$ is one-to-one [6, Theorem 2.1] and carries convolution into pointwise multiplication. Furthermore the analytic Feyman integral exists for every $F$ in $S$ [6, Theorem 5.1].

Theorem 3.1. Assume that for s-a.e. $x \in C_{1}[0, T], F(x)$ is given by (1.3) where $\left\{A\left(s_{1}, \cdots, a_{n}\right)=\left(a_{i j}\left(s_{1}, \cdots, s_{n}\right)\right): i, j=1,2, \cdots, n,\left(s_{1}, \cdots, s_{n}\right) \in Q\right\}$ is a commutative family of $n$ by $n$ real, symmetric, nonnegative definite matrices such that the nonnegative eigenvalues $p_{1}\left(s_{1}, \cdots, s_{n}\right) \cdots, p_{n}\left(s_{1}, \cdots, s_{n}\right)$ are each elements of $L_{1}(Q)$. Then $F$ is in the Banach algebra $S$ and hence is analytic Feynman integrable.

Proof. We seek an element $\sigma$ in $M\left(L_{2}[0, T]\right)$ such that for each $\rho>0$,

$$
F(\rho x)=\int_{L_{2}[0, T]} \exp \left\{i \rho \int_{0}^{T} g(s) \tilde{d} x(s)\right\} d \sigma(g)
$$

for $m_{1}$-a.e. $x$ in $C_{1}[0, T]$. Let $B=\left(b_{i_{j}}\right)$ be an orthogonal matrix such that $B A\left(s_{1}, \cdots, s_{n}\right) B^{-1}=P\left(s_{1}, \cdots, s_{n}\right)$ throughout $Q$ where $P\left(s_{1}, \cdots, s_{n}\right)$ is a diagonal matrix with nonnegative entries $p_{1}\left(s_{1}, \cdots, s_{n}\right), \cdots, p_{n}\left(s_{1}, \cdots, s_{n}\right) \in$ $L_{1}(Q)$.

Let $\rho>0$ be given. Then for $m_{1}$-a.e. $x \in C_{1}[0, T]$, we have

$$
\begin{aligned}
& F(\rho x) \\
= & \exp \left\{-\rho^{2} \int_{Q}\left\langle B^{-1} P B\left(x\left(s_{1}\right), \cdots, x\left(s_{n}\right)\right),\left(x\left(s_{1}\right), \cdots, x\left(s_{n}\right)\right)\right\rangle d s_{1} \cdots d s_{n}\right\} \\
= & \exp \left\{-\rho^{2} \int_{Q} \sum_{j=1}^{n} p_{j}\left(s_{1}, \cdots, s_{n}\right)\left[\sum_{k=1}^{n} b_{j k} x\left(s_{k}\right)\right]^{2} d s_{1} \cdots d s_{n}\right\} \\
= & \exp \left\{-\frac{1}{2} \sum_{j=1}^{n} \int_{Q}\left[\rho \sqrt{2} \sum_{k=1}^{n} b_{j k}\left(p_{j}\left(s_{1}, \cdots, s_{n}\right)\right)^{1 / 2} x\left(s_{k}\right)\right]^{2} d s_{1} \cdots d s_{n}\right\}
\end{aligned}
$$




$$
\begin{gathered}
=\int_{C_{n}(Q)} \cdots \int_{C_{n}(Q)} \exp \left\{\sum _ { j = 1 } ^ { n } \left(i \rho \sqrt{2} \int_{Q}\left[\sum_{k=1}^{n} b_{j k}\left(p_{j}\left(s_{1}, \cdots, s_{n}\right)\right)^{1 / 2} x\left(s_{k}\right)\right]\right.\right. \\
\left.\left.\tilde{d}_{n} f_{j}\left(s_{1}, \cdots, s_{n}\right)\right)\right\} d m_{n}\left(f_{1}\right) d m_{n}\left(f_{2}\right) \cdots d m_{n}\left(f_{n}\right) \\
=\int_{C_{n}(Q)} \cdots \int_{C_{n}(Q)} \exp \left\{i \rho \sqrt { 2 } \sum _ { k = 1 } ^ { n } \sum _ { j = 1 } ^ { n } \left(\int_{0}^{T}\left[\int_{E_{k}\left(s_{k}\right)} b_{j k}\left(p_{j}\left(t_{1}, \cdots, t_{n}\right)\right)^{1 / 2} \tilde{d}_{n} f_{j}\left(t_{1}, \cdots, t_{n}\right)\right]\right.\right. \\
\left.\left.\tilde{d} x\left(s_{k}\right)\right)\right\} d m_{n}\left(f_{1}\right) \cdots d m_{n}\left(f_{n}\right),
\end{gathered}
$$

where we have made use of Theorem 2.1.

Next we define $\mathscr{T}:\left[C_{n}(Q)\right]^{n} \rightarrow L_{2}[0, T]$ by the formula

$$
\mathscr{T}\left(f_{1}, \cdots, f_{n}\right)(s)=\sqrt{2} \sum_{k=1}^{n} \sum_{j=1}^{n} b_{j k} \int_{E_{k}(s)}\left(p_{j}\left(t_{1}, \cdots, t_{n}\right)\right)^{1 / 2} \tilde{d}_{n} f_{j}\left(t_{1}, \cdots, t_{n}\right) .
$$

We need to show that $\mathscr{T}$ is a Borel measurable function of $\left(s, f_{1}, \cdots, f_{n}\right)$ on $[0, T] \times\left[C_{n}(Q)\right]^{n}$ and that for $m_{n} \times m_{n} \times \cdots \times m_{n}-$ a.e. $\left(f_{1}, \cdots, f_{n}\right)$ in $\left[C_{n}(Q)\right]^{n}, \mathscr{T}\left(f_{1}, \cdots, f_{n}\right)(\cdot)$ is in $L_{2}[0, T]$. But these facts will follow quite readily once we show that each term

$g_{j k}\left(s, f_{j}\right)=\int_{E_{k}(s)}\left[p_{j}\left(t_{1}, \cdots, t_{n}\right)\right]^{1 / 2} \tilde{d}_{n} f_{j}\left(t_{1}, \cdots, t_{n}\right)=\int_{Q} \sqrt{p_{j}} \chi_{[s, T]}\left(t_{k}\right) \tilde{d}_{n} f_{j}\left(t_{1}, \cdots, t_{n}\right)$

on the right hand side of (3.2) satisfies properties (a) and (b) below:

(a) $g_{j k}\left(s, f_{j}\right)$ is a Borel measurable function of $\left(s, f_{j}\right)$ on $[0, T] \times C_{n}(Q)$.

(b) For s-a.e. $f_{j}$ in $C_{n}(Q), g_{j k}\left(s, f_{j}\right)$ is in $L_{2}[0, T]$.

Proof of claim (a). Let $\left\{\phi_{j}\right\}$ be a CON set of functions of bounded variation on $Q$. Let $\Phi(s)\left(t_{1}, \cdots, t_{n}\right):[0, T] \rightarrow L_{2}(Q)$ be given by

$$
\Phi(s)\left(t_{1}, \cdots, t_{n}\right)=\left[p_{j}\left(t_{1}, \cdots, t_{n}\right)\right]^{1 / 2} \chi_{[s, T]}\left(t_{k}\right) .
$$

Then, $\Phi$ is continuous and hence Borel measurable. Also

$$
\begin{aligned}
g_{j k}\left(s, f_{j}\right) & =\int_{Q} \Phi(s)\left(t_{1}, \cdots, t_{n}\right) \tilde{d}_{n} f_{j}\left(t_{1}, \cdots, t_{n}\right) \\
& =\lim _{m \rightarrow \infty} \sum_{i=1}^{m}\left(\Phi(s), \phi_{i}\right) \int_{Q} \phi_{i}\left(t_{1}, \cdots, t_{n}\right) d f_{j}\left(t_{1}, \cdots, t_{n}\right) .
\end{aligned}
$$

It is easy to see that each term on the right hand side of (3.3) is a continuous and hence Borel measurable function of $\left(s, f_{j}\right)$ on $[0, T] \times C_{n}(Q)$ and so $g_{j k}\left(s, f_{j}\right)$ is Borel measurable.

Proof of claim (b). This follows from the Fubini Theorem: 


$$
\begin{aligned}
\int_{C_{n}(Q)} & \left(\int_{0}^{T} g_{j k}^{2}\left(s, f_{j}\right) d s\right) d m_{n}\left(f_{j}\right) \\
& =\int_{0}^{T}\left(\int_{C_{n}(Q)} g_{j k}^{2}\left(s, f_{j}\right) d m_{n}\left(f_{j}\right)\right) d s \\
& =\int_{0}^{T}\left\|\left[p_{j}(\cdot, \cdots, \cdot)\right]^{1 / 2} \chi_{E_{k}(s)}(\cdot, \cdots, \cdot)\right\|_{2}^{2} d s \\
& \leq T\left\|p_{j}\right\|_{1}<\infty .
\end{aligned}
$$

Thus we arrive at the following expression for $m_{1}$-a.e. $x \in C_{1}[0, T]$ :

$$
\begin{aligned}
F(\rho x) & =\int_{C_{n}(Q)} \cdots \int_{C_{n}(Q)} \exp \left\{i \rho \int_{0}^{T} \mathscr{T}\left(f_{1}, \cdots, f_{n}\right)(s) \tilde{d} x(s)\right\} d m_{n}\left(f_{1}\right) \cdots d m_{n}\left(f_{n}\right) \\
& =\int_{L_{2}[0, T]} \exp \left\{i \rho \int_{0}^{T} g(s) \tilde{d} x(s)\right\} d \sigma(g)
\end{aligned}
$$

with $\sigma \equiv\left[m_{n}\right]^{n} \circ \mathscr{T}^{-1} \in M\left(L_{2}[0, T]\right)$. This expression for $F$ implies that $F$ is in the Banach algebra $S$, which concludes the proof of Theorem 3.1.

Next we develop several corollaries to Theorem 3.1. Our first corollary is the main result in [8].

Corollary 3.1. Theorem 4.1 of [8]. This is the case $n=2$ and the eigenvalues $p_{1}\left(s_{1}, s_{2}\right)$ and $p_{2}\left(s_{1}, s_{2}\right)$ have square roots which are of bounded variation on $[0, T]^{2}$.

In order to apply Theorem 3.1 to functions $F$ of the form (1.3) one needs to compute the $n$ eigenvalues $p_{1}\left(s_{1}, \cdots, s_{n}\right), \cdots, p_{n}\left(s_{1}, \cdots, s_{n}\right)$ and show that they are in $L_{1}(Q)$. In our next corollary we put the hypothesis directly on the functions $a_{i j}\left(s_{1}, \cdots, s_{n}\right)$ rather than on the eigenvalues. The hypotheses of this corollary are very simple as compared to those of the corresponding corollary in [8, Corollary 4.2].

CoRollary 3.2. Let $F(x)$ be of the form (1.3) where the matrices $\left\{A\left(s_{1}, \cdots, s_{n}\right)=\left(a_{i j}\left(s_{1}, \cdots, s_{n}\right)\right): i, j=1,2, \cdots, n\right\}$ are a commutative family of $n$ by $n$ real, symmetric, positive definite matrices with the functions $a_{i j}\left(s_{1}, \cdots, s_{n}\right)$ all in $L_{1}(Q)$. Then the conclusions of Theorem 3.1 hold.

Proof. Since $B A\left(s_{1}, \cdots, s_{n}\right) B^{-1}=P\left(s_{1}, \cdots, s_{n}\right)$, a diagonal matrix with the eigenvalues $p_{1}\left(s_{1}, \cdots, s_{n}\right), \cdots, p_{n}\left(s_{1}, \cdots, s_{n}\right)$ along the diagonal, we see that each eigenvalue $p_{j}\left(s_{1}, \cdots, s_{n}\right)$ is a finite linear combination of the functions $a_{i j}\left(s_{1}, \cdots, s_{n}\right)$ and so is in $L_{1}(Q)$.

Corollary 3.3. Let $F(x)$ be of the form (1.3) with $A\left(s_{1}, \cdots, s_{n}\right)=$ $g\left(s_{1}, \cdots, s_{n}\right) A$ where $g\left(s_{1}, \cdots, s_{n}\right) \geq 0$ is in $L_{1}(Q)$ and $A$ is a real, constant, 
symmetric and nonnegative definite matrix. Then the conclusions of Theorem 3.1 hold since the eigenvalues of $A\left(s_{1}, \cdots, s_{n}\right)$ are simply the eigenvalues of $A$ multiplied by the $L_{1}$-function $g\left(s_{1}, \cdots, s_{n}\right)$.

In our next corollary we show that a broad class of functions belong to the Banach algebra $S$.

Corollary 3.4. Let $\left\{A\left(s_{1}, \cdots, s_{n}\right)\right\}$ be as in Theorem 3.1. Let $\eta$ be a Borel measure on $Q=[0, T]^{n}$. Let $\theta: Q \times \boldsymbol{R}^{n} \rightarrow C$ be such that for all $\left(s_{1}, \cdots, s_{n}\right) \in Q$,

$$
\theta\left(s_{1}, \cdots, s_{n} ; u_{1}, \cdots, u_{n}\right)=\int_{R_{n}} \exp \left\{i \sum_{j=1}^{n} u_{j} v_{j}\right\} d \sigma_{\left(s_{1}, \cdots, s_{n}\right)}\left(v_{1}, \cdots, v_{n}\right)
$$

where

i) $\sigma_{\left(s_{1}, \cdots, s_{n}\right)} \in M\left(\boldsymbol{R}^{n}\right)$,

ii) for all $E \in \mathscr{B}\left(\boldsymbol{R}^{n}\right), \sigma_{\left(s_{1}, \ldots, s_{n}\right)}(E)$ is a Borel measurable function of $\left(s_{1}, \cdots, s_{n}\right)$,

iii) $\left\|\sigma_{\left(s_{1}, \cdots, s_{n}\right)}\right\| \in L_{1}(Q, \mathscr{B}(Q), \eta)$.

Then the function

$$
\begin{aligned}
& G(x) \\
& =\exp \left\{-\int_{Q}\left\langle A\left(s_{1}, \cdots, s_{n}\right)\left(x\left(s_{1}\right), \cdots, x\left(s_{n}\right)\right),\left(x\left(s_{1}\right), \cdots, x\left(s_{n}\right)\right)\right\rangle d s_{1} \cdots d s_{n}\right. \\
& \left.\quad+\int_{Q} \theta\left(s_{1}, \cdots, s_{n} ; x\left(s_{1}\right) \cdots, x\left(s_{n}\right)\right) d \eta\left(s_{1}, \cdots, s_{n}\right)\right\}
\end{aligned}
$$

belongs to $S$.

Proof. Let $G_{1}(x)=\exp \left\{\int_{Q} \theta\left(s_{1}, \cdots, s_{n} ; x\left(s_{1}\right), \cdots, x\left(s_{n}\right)\right) d \eta\left(s_{1}, \cdots, s_{n}\right)\right\}$. Then $G(x)=F(x) G_{1}(x)$ where $F(x)$ is given by (1.3). By Theorem 3.1 above, $F$ belongs to $S$ and by Corollary 4 of [10], $G_{1}(x)$ belongs to $S$. Since $S$ is a Banach algebra we have $G \in S$.

\section{\$4. The ע-dimensional theory}

Let $\nu$ be a positive integer and let $C_{1}^{\nu}[0, T]$ denote $\nu$-dimensional Wiener space; that is the set of $R^{\nu}$-valued continuous functions $\vec{X}=$ $\left(x_{1}, \cdots, x_{\nu}\right)$ on $[0, T]$ such that $\vec{X}(0)=0$. Let $m_{1}^{\nu}$ denote Wiener measure on $C_{1}^{\nu}[0, T]$ and let $M\left(L_{2}^{\nu}[0, T]\right)$ be the collection of complex-valued countably additive measures on $\mathscr{B}\left(L_{2}^{\nu}[0, T]\right)$. The Banach algebra $S(\nu)$ consists of functions $F$ on $C_{1}^{\nu}[0, T]$ expressible in the form 


$$
F(\vec{X})=\int_{L_{L}^{\nu}[0, T]} \exp \left[i \sum_{k=1}^{\nu} \int_{0}^{T} v_{k}(t) \tilde{d} x_{k}(t)\right] d \sigma\left(v_{1}, \cdots, v_{\nu}\right)
$$

for s-a.e. $\vec{X}$ in $C_{1}^{\nu}[0, T]$, where $\sigma$ is an element of $M\left(L_{2}^{\nu}[0, T]\right)$.

In this section, as mentioned birefly in the introduction, we extend the results of section 3 to $C_{1}^{\nu}[0, T]$. We first give a $\nu$-dimensional version of Theorem 3.1.

Theorem 4.1. Let $n$ and $\nu$ be positive integers. Assume that for sa.e. $\vec{X}$ in $C_{1}^{\nu}[0, T]$,

$$
\begin{aligned}
F(\vec{X})= & \exp \left\{-\int_{0}^{T} \cdots \int_{0}^{T}\left\langle A\left(s_{1}, \cdots, s_{n}\right)\left(\vec{X}\left(s_{1}\right), \cdots, \vec{X}\left(s_{n}\right)\right),\right.\right. \\
& \left.\left.\left(\vec{X}\left(s_{1}\right), \cdots, \vec{X}\left(s_{n}\right)\right)\right\rangle d s_{1} \cdots d s_{n}\right\}
\end{aligned}
$$

where $\left\{A\left(s_{1}, \cdots, s_{n}\right)=\left(a_{i j}\left(s_{1}, \cdots, s_{n}\right)\right): i, j=1,2, \cdots, \nu n,\left(s_{1}, \cdots, s_{n}\right) \in Q\right\}$ is a commutative family of $\nu$ by $\nu$ n real, symmetric, nonnegative definite matrices such that the (nonnegative) eigenvalues $p_{1}\left(s_{1}, \cdots, s_{n}\right), \cdots, p_{\nu n}\left(s_{1}\right.$, $\left.\cdots, s_{n}\right)$ are each elements of $L_{1}(Q)$. Then $F$ is in the Banach algebra $S(\nu)$. Furthermore $F$ is analytic Feynman integrable.

Outline of Proof. We may assume that both $n$ and $\nu$ are greater than 1 since the case $\nu=1$ and general $n$ is Theorem 3.1 above, while the case $n=1$ and general $\nu$ is the main result of [19]. We will omit the measurability arguments; they are similar to arguments given eariler in this paper. In fact we will simply give a brief outline of the key calculations. These calculations however are quite complicated.

For $r \in \boldsymbol{R}$ let $h[r]$ denote the greatest integer less than or equal to $r$. Let $\rho>0$ be given. Let $B=\left(b_{j, k}\right)$ be an $\nu n$ by $\nu n$ orthogonal matrix such that $B A\left(s_{1}, \cdots, s_{n}\right) B^{-1}=P\left(s_{1}, \cdots, s_{n}\right)$ throughout $Q$ where $P\left(s_{1}, \cdots, s_{n}\right)$ is an $\nu n$ by $\nu n$ diagonal matrix with nonnegative entries $p_{1}\left(s_{1}, \cdots, s_{n}\right), \cdots$, $p_{\nu n}\left(s_{1}, \cdots, s_{n}\right)$, the eigenvalues of $A\left(s_{1}, \cdots, s_{n}\right)$. Then for $m_{1}^{\nu}$-a.e. $\vec{X}$ in $C_{1}^{\nu}[0, T]$ we obtain that

$$
\begin{aligned}
& F(\rho \vec{X})= \exp \left[-\rho^{2} \int_{Q}\left\langle P\left(s_{1}, \cdots, s_{n}\right) B\left(\vec{X}\left(s_{1}\right), \cdots, \vec{X}\left(s_{n}\right)\right), B\left(\vec{X}\left(s_{1}\right), \cdots, \vec{X}\left(s_{n}\right)\right)\right\rangle\right. \\
&\left.d s_{1} \cdots d s_{n}\right] \\
&=\sum_{j=1}^{\nu n} \exp \left[-\rho^{2} \int_{Q}\left\{\sum_{k=1}^{\nu n} b_{j, k}\left[p_{j}\left(s_{1}, \cdots, s_{n}\right)\right]^{1 / 2} x_{1+(k-1) \bmod (\nu)}\left(s_{1+h[(k-1) / \nu]}\right)\right\}^{2}\right. \\
&\left.d s_{1} \cdots d s_{n}\right]
\end{aligned}
$$




$$
\begin{gathered}
=\int_{C_{n}(Q)} \cdots \int_{C_{n}(Q)} \exp \left[i \rho \sqrt { 2 } \sum _ { k = 1 } ^ { \nu n } \sum _ { j = 1 } ^ { \nu n } \int _ { Q } \left\{b_{j, k}\left[p_{j}\left(s_{1}, \cdots, s_{n}\right)\right]^{1 / 2} x_{1+(k-1) \bmod (\nu)}\right.\right. \\
\left.\left.\left(s_{1+h[(k-1) / \nu]}\right)\right\} \tilde{d}_{n} f_{j}\left(s_{1}, \cdots, s_{n}\right)\right] d m_{n}\left(f_{1}\right) d m_{n}\left(f_{2}\right) \cdots d m_{n}\left(f_{\nu n}\right) \\
=\int_{C_{n}(Q)} \cdots \int_{C_{n}(Q)} \exp \left[i \rho \sqrt { 2 } \sum _ { k = 1 } ^ { \nu n } \sum _ { j = 1 } ^ { \nu n } \int _ { 0 } ^ { T } \left\{\int_{J_{k}} b_{j, k}\left[p_{j}\left(t_{1}, \cdots, t_{n}\right)\right]^{1 / 2}\right.\right. \\
\left.\left.\tilde{d}_{n} f_{j}\left(t_{1}, \cdots, t_{n}\right)\right\} \tilde{d} x_{1+(k-1 \bmod (\nu)}\left(s_{1+h[(k-1) / \nu]}\right)\right] d m_{n}\left(f_{1}\right) \cdots d m_{n}\left(f_{\nu n}\right)
\end{gathered}
$$

where the last equality follows from Theorem 2.1 and $J_{k}$ denotes the set $E_{1+h[(k-1) / \nu]}\left(s_{1-h[(k-1) / \nu]}\right)$; see Theorem 2.1 for the definition of $E_{k}\left(s_{k}\right)$.

Next for $\alpha=1,2, \cdots, \nu$ let

$$
\mathscr{T}_{\alpha}\left(f_{1}, \cdots, f_{\nu n}\right)(s)=\sqrt{2} \sum_{m=0}^{n-1} \sum_{j=1}^{\nu n} \int_{E_{m+1}(s)} b_{j, \nu m+\alpha}\left[p_{j}\left(t_{1}, \cdots, t_{n}\right)\right]^{1 / 2} \tilde{d}_{n} f_{j}\left(t_{1}, \cdots, t_{n}\right) .
$$

Then

$F(\rho \vec{X})=\int_{C_{n}(Q)} \cdots \int_{C_{n}(Q)} \exp \left[i \rho \sum_{\alpha=1}^{\nu} \int_{0}^{T} \mathscr{T}_{\alpha}\left(f_{1}, \cdots, f_{\nu n}\right)(s) \tilde{d} x_{\alpha}(s)\right] d m_{n}\left(f_{1}\right) \cdots d m_{n}\left(f_{\nu n}\right)$.

Now let $\mathscr{T}:\left[C_{n}(Q)\right]^{\nu n} \rightarrow L_{2}^{\nu}[0, T]$ be defined by the formula $\mathscr{T}\left(f_{1}, \cdots, f_{\nu n}\right)(\cdot)$ $=\left(\mathscr{T}_{1}\left(f_{1}, \cdots, f_{\nu n}\right)(\cdot), \cdots, \mathscr{T}_{\nu}\left(f_{1}, \cdots, f_{\nu n}\right)(\cdot)\right)$, and let $\sigma=\left[m_{n}\right]^{\nu n} \circ \mathscr{T}^{-1}$. Then $\sigma$ is an element of $M\left(L_{2}^{\nu}[0, T]\right)$ and for $m_{1}^{\nu}$-a.e. $\vec{X}$ in $C_{1}^{\nu}[0, T]$ we have, using the change of variables theorem [14, p. 163], that

$$
F(\rho \vec{X})=\int_{L_{2}^{\nu}[0, T]} \exp \left[i \rho \sum_{\alpha=1}^{\nu} \int_{0}^{T} v_{\alpha}(t) \tilde{d} x_{\alpha}(t)\right] d \sigma\left(v_{1}, \cdots, v_{\nu}\right) .
$$

Thus $F$ is an element of $S(\nu)$ which completes the proof of Theorem 4.1.

Remark. Note that Theorem 4.1 has many corollaries; for example the main result of [8], the main result of [19] and Theorem 3.1 above. In addition, Corollaries 3.2-3.4 above all have $\nu$-dimensional counterparts which are quite straightforward to formulate and hence precise statements of them will not be given.

\section{§ 5. Fresnel integrals}

In this section we show that functions of the form (1.3) or (3.5) with an appropriate slight modification are Fresnel integrable. Let $H$ be the space of absolutely continuous functions $\gamma$ on $[0, T]$ which vanish at $T$ and whose derivatives $D \gamma$ are in $L_{2}[0, T] . \quad H$ is a Hilbert space under the inner product 


$$
\left(\gamma_{1}, \gamma_{2}\right)=\int_{0}^{T}\left(D \gamma_{1}\right)(s)\left(D \gamma_{2}\right)(s) d s
$$

Albeverio and Hфegh-Krohn's space $\mathscr{F}(H)$ of Fresnel integrable functions consists of Fourier transforms of finite Borel measures on $H[1]$. The spaces $\mathscr{F}(H)$ and $S$ are isometrically isomorphic as Banach algebras as was shown by Johnson [15].

Theorem 5.1. For each $\gamma$ in $H$ let

$$
\begin{gathered}
G(\gamma)=\exp \left\{-\int_{0}^{T} \cdots \int_{0}^{T}\left\langle A\left(s_{1}, \cdots, s_{n}\right)\left(\gamma\left(s_{1}\right)-\gamma(0), \cdots, \gamma\left(s_{n}\right)-\gamma(0)\right),\right.\right. \\
\left.\left(\gamma\left(s_{1}\right)-\gamma(0), \cdots, \gamma\left(s_{n}\right)-(0)\right)>d s_{1} \cdots d s_{n}\right\}
\end{gathered}
$$

where the matrices $\left\{A\left(s_{1}, \cdots, s_{n}\right)\right\}$ are as in Theorem 3.1. Then $G$ is in $\mathscr{F}(H)$.

Proof. This theorem follows from Theorem 3.1 above and ideas from [15]. The key idea is that equation (2.9) holds for s-a.e. $f$ in $C_{n}(Q)$ for each $x$ in $C_{1}[0, T]$ which is absolutely continuous and whose derivative is in $L_{2}[0, T]$. This, in turn, implies that equation (3.4) holds for each such $x$.

Finally, using Theorem 5.1, a result from [9], and the fact that $\mathscr{F}(H)$ is a Banach algebra we obtain the following corollary.

Corollary 5.1. Let $\left\{A\left(s_{1}, \cdots, s_{n}\right)\right\}, \eta$ and $\theta$ be as in Corollary 3.4. For $\gamma$ in $H$ let

$G_{1}(\gamma)=\exp \left\{\int_{0}^{T} \cdots \int_{0}^{T} \theta\left(s_{1}, \cdots, s_{n} ; \gamma\left(s_{1}\right)-\gamma(0), \cdots, \gamma\left(s_{n}\right)-\gamma(0)\right) d \eta\left(s_{1}, \cdots, s_{n}\right)\right\}$.

Then the functions $G_{1}(\gamma)$ and $G_{1}(\gamma) G(\gamma)$, where $G$ is given by equation (5.1), are in the Banach algebra $\mathscr{F}(H)$.

Remark. Proceeding as in section 4 above it is quite easy to see that Theorem 5.1 and Corollary 5.1 also have $\nu$-dimensional counterparts.

\section{REFERENCES}

[1] S. Albeverio and R. Høegh-Krohn, Mathematical theory of Feynman path integrals, Springer Lecture Notes in Mathematics, Berlin, 523 (1976).

[2] - Oscillatory integrals and the method of stationary phase in infinitely many dimensions, with applications to the classical limit of quantum mechanics, I, Invent. Math., 40 (1977), 59-106.

[ 3 ] — - Feynman path integrals and the corresponding method of stationary phase, 
in Feynman Path Integrals, Marseille, 1978, Springer Lecture Notes in Physics, 106 (1979), 3-57.

[4] E. Berkson and T. A. Gillespie, Absolutely continuous functions of two variables and well-bounded operators, J. London Math. Soc., 30 (1984), 305-321.

[5] R. H. Cameron and D. A. Storvick, An operator valued function space integral applied to multiple integrals of functions of class $L_{1}$, Nagoya Math. J., 51 (1973), 91-122.

[6] - - Some Banach algebras of analytic Feynman integrable functionals, in Analytic Functions, Kozubnik, 1979, Springer Lecture Notes in Mathematics, Berlin, 798 (1980), 18-67.

[7] K. S. Chang, Scale-invariant measurability in Yeh-Wiener space, J. of Korean Math. Soc., 19 (1982), 61-67.

[8] K. S. Chang, G. W. Johnson and D. L. Skoug, The Feynman integral of quadratic potentials depending on two time variables, Pacific J. Math., 122 (1986), 11-33.

[9] - Functions in the Fresnel class, Proc. Amer. Math. Soc., 100 (1987), 309-318.

[10] - Functions in the Banach algebra $S(\nu)$, J. of Korean Math. Soc., 24 (1987), 151-158.

[11] D. Elworthy and A. Truman, Feynman maps, Cameron-Martin formulae and anharmonic oscillators, Ann. Inst. H. Poincaré, 41 (1984), 115-142.

[12] R. J. Feynman, Space-time approach to non-relativistic quantum mechanics, Rev. Mod. Phys., 20 (1948), 367-387.

[13] R. J. Feynman and A. R. Hibbs, Quantum Mechanics and Path Integrals, McGrawHill, New York, 1965.

[14] F. Halmos, Measure Theory, Van-Nostrand, Princeton, New Jersey, 1950.

[15] G. W. Johnson, The equivalence of two approaches to the Feynman integral, J. Math. Phys., 23 (1982), 2090-2096.

[16] G. W. Johnson and D. L. Skoug, A Banach algebra of Feynman integrable functionals with applications to an integral equation formally equivalent to Schroedinger's equation, J. Funct. Anal., 12 (1973), 129-152.

[17] — Scale-invariant measurability in Wiener space, Pacific J. Math., 83 (1979), 157-176.

[18] —- Notes on the Feynman integral, I, Pacific J. Math., 93 (1981), 313-324.

[19] —- Notes on the Feynman integral, II, J. Funct. Anal., 41 (1981), 277-289.

[20] - Notes on the Feynman integral, III, The Schroedinger equation, Pacific J. Math., 105 (1983), 321-358.

[21] E. Nelson, The use of the Wiener process in quantum theory, preprint intended for inclusion in Vol. III of the Collected Works of Norbert Wiener, edited by P. Masani, M.I.T. Press.

\author{
Chull Park \\ Department of Mathematics \\ and Statistics \\ Miami University \\ Oxford, $\mathrm{OH} 45056$ \\ David Skoug \\ Department of Mathematics \\ and Statistics \\ University of Nebraska \\ Lincoln, NE 68588-0323
}

